\title{
Efeitos da resolução espacial do modelo VIC na previsão de vazão diária a usinas hidroelétricas do Rio Uruguai
}

\section{Effects of spatial resolutions of model VIC on the daily inflow forecasts to hydropower plant of the Uruguay River basin}

Data de entrada: $05 / 12 / 2019$

Data de aprovação: $18 / 03 / 2020$

Vinícius Siqueira Oliveira Carvalho ${ }^{1}$ | José Anderson do Nascimento Batista ${ }^{1 *}$ | Paulo Sergio Franco Barbosa ${ }^{1}$ | Vinícius de Carvalho Neiva Pinheiro' ${ }^{1}$ | Alberto Luiz Francato'

DOI: https://doi.org/10.36659/dae.2021.057

ORCID ID

Carvalho VSO (D) https://orcid.org/0000-0002-8209-3890

Batista JAN (iD https://orcid.org/0000-0001-9041-2436
Barbosa PSF (D) https://orcid.org/0000-0003-4287-0816

Pinheiro VCN (D) https://orcid.org/0000-0002-4049-7256

Francato AL (D) https://orcid.org/0000-0002-5815-8068

\section{Resumo}

A geração elétrica brasileira é amplamente dependente das usinas hidroelétricas. Este estudo tem objetivo de verificar o desempenho de diferentes resoluções espaciais do modelo hidrológico semi-distribuído Variable Infiltration Capacity (VIC) acoplado ao modelo meteorológico numérico Eta, este adotado com resolução espacial fixa em 15 km, para a previsão de vazão diária de cinco usinas do Rio Uruguai, no sul do Brasil. 0 modelo hidrológico foi aplicado para as resoluções $0,04^{\circ}, 0,08^{\circ}$ e $0,16^{\circ}$, em que cada célula de grade contém propriedades uniformes de solo, relevo e vegetação. As usinas hidroelétricas de jusante da área de estudo apresentaram melhor desempenho de previsão de vazão diária pelo modelo VIC para as resoluções mais finas. Conforme o Inventário de Restrições Operativas Hidráulicas dos Aproveitamentos Hidroelétricos (ROH) do Operador Nacional do Sistema (ONS), o efeito da resolução espacial do modelo VIC também foi estudado quanto ao desempenho da previsão diária de riscos de cheias, a partir de matrizes de contingências. Nos picos de cheias, a sensibilidade do modelo a valores acima de cada restrição de segurança (atenção, alerta e emergência) mantém-se invariável igual a 100\%, enquanto a precisão variou de 51 a 100\%, ambas sem tendência significativa em relação à resolução espacial. Todas as usinas mostraram acurácia mínima de $98,3 \%$ e de forma crescente em relação à resolução espacial do modelo. A heterogeneidade de dados físicos foi fator limitante para os ganhos do refinamento da resolução espacial do modelo hidrológico.

Palavras-chave: Modelo VIC. Previsão de vazão. Rio Uruguai. Matrizes de contingência.

\section{Abstract}

The Brazilian national electric power generation is widely dependent on the hydroelectric dams. This study aims to assess the performance of different space resolutions of the hydrological semi-distributed model Variable Infiltration Capacity (VIC) coupled to the numerical weather model Eta, this steadily used with $15 \mathrm{~km}$ in space resolution, to the daily inflow prediction of five reservoirs at the upper Uruguay river, in southern Brazil. The hydrological model was applied to the resolutions of $0.04^{\circ}, 0.08^{\circ}$ and $0.16^{\circ}$, where each grid cell contains uniform soil, relief and vegetation properties. The downstream dams showed higher performances to the daily inflow

\footnotetext{
${ }^{1}$ Universidade Estadual de Campinas - Campinas - São Paulo - Brasil.

* Autor correspondente: nbatistaaunicamp.br.
} 
predictions with the model VIC for finer resolutions. According to the Brazilian System National Operator's Inventory of Hydraulic Operative Restrictions of Hydroelectric Harnessing, the effect of VIC's resolution over the daily flood risk forecast performance was assessed throughout the contingency matrix. The model sensibility to streamflow above each safety constrain (attention, alert and emergency) stands still equal to $100 \%$, while the precision varied within 51 to $100 \%$, both without any clear trend with respect to the model resolution. Every dam showed minimum accuracy of $98.3 \%$ in such a way that it increases with respect to the model spatial resolution. The heterogeneity in the physical data was a limiting factor to the gains from the spatial resolution refinement of the hydrological model.

Keywords: VIC model. Inflow forecast. Uruguay River. Contingency matrix.

\section{INTRODUÇÃO}

De acordo com Dalseno et al. (2017), nos últimos dez anos, em média, a energia hidrelétrica forneceu $85 \%$ da oferta total de energia elétrica no Brasil; entre 2000 e 2012, a contribuição foi de $91 \%$ da oferta; no entanto, de 2013 a 2016, a participação caiu para 75\%. Dados do Anuário Estatístico de Energia Elétrica de 2018 da Empresa de Pesquisa Energética (EPE) indicam que a participação das fontes hidráulicas na geração elétrica nacional corresponde a $63 \%$. Entretanto, a energia elétrica proveniente dos recursos hídricos é ainda a fonte de maior potencial energético instalado no país.

O Operador Nacional do Sistema (ONS) dispõe de modelos de previsão que usam a precipitação observada e prevista como variáveis de entrada, tanto para previsões diárias, as quais são importantes para operação em tempo real, como para previsões mensais, indispensáveis para planejamento e operação do sistema hidroelétrico (COLLISCHONN et al., 2007). No Brasil, usinas hidrelétricas (UHE) utilizam modelos de previsão baseados de forma simplificada apenas nas precipitações e vazões observadas, como o modelo PREVIVAZ, sem representar sistematicamente a participação dos processos hidrológicos envolvidos. O modelo PREVIVAZ (GUILHON et al., 2007) é um modelo baseado na desagregação de previsões semanais a partir da tendência inferida a partir apenas de vazões antecedentes.

Em outra mão aos modelos estocásticos, os modelos distribuídos permitem representar de maneira conjunta os processos hidrológicos envolvidos e a espacialização de tais processos (ANDERSEN et al., 2002; HADDELAND et al., 2002; LIANG et al., 2004). Os processos ambientais apresentam comportamentos não lineares, devido à alta complexidade e ao elevado dinamismo. Por esta razão, os modelos hidrológicos distribuídos são mais sensíveis a heterogeneidades espaciais e temporais tanto em dados meteorológicos como em dados hidrológicos e geográficos, como tipo de solo, cobertura vegetal e topografia (BEVEN, 2001; HADDELAND et al., 2002; LIANG et al., 2004). Além disso, modelos distribuídos representam de maneira tão apropriada quanto possível a separação do escoamento superficial, o qual chega mais rápido ao reservatório, e do escoamento subterrâneo, o qual demora mais para atingir os cursos d'água e reservatórios (COLLISCHONN et al., 2007; MOREDA et al., 2006).

O modelo hidrológico distribuído $M G B$, sigla para Modelo de Grandes Bacias (COLLISCHONN et al., 2010; COLLISCHONN; TUCCI, 2001), encontra-se implementado nas UHE de Itumbiara e São Simão, rio Paranaíba (COLLISCHONN 
et al., 2007), e nas usinas de Campos Novos e Barra Grande, Rio Uruguai (FAN et al., 2017). Em ambos os estudos, as precipitações foram adotadas a partir de previsões obtidas pelo modelo numérico meteorológico Eta (CHOU et al., 2000; MESINGER et al., 1988).

Na bacia do Paraná, Collischonn et al. (2007) compararam as previsões provenientes do MGB com as previsões obtidas pelo PREVIVAZ. O modelo distribuído apresentou melhor desempenho estatístico, comportando-se de maneira eficaz nos períodos seco e úmido. Já no estudo realizado por Fan et al. (2017) na UHE Campos Novos, os resultados mostraram desempenho adequado para quase todas as previsões que antecedem a cheia. Por outro lado, na UHE Barra Grande, as previsões de vazões apresentaram melhor comportamento apenas com previsão de 2 a 3 dias antes da ocorrência do evento extremo. Embora ambas as usinas estejam localizadas na cabeceira da bacia do Rio Uruguai, as áreas de drenagem de ambas possuem respostas hidrológicas diferentes.

A atual disponibilidade de dados provenientes das técnicas de sensoriamento remoto, em resolução compatível com bacias hidrográficas de usinas hidroelétricas, tem ampliado o uso de modelos hidrológicos distribuídos para a previsão de vazão. Por outro lado, a resolução espacial do balanço hídrico desses modelos ainda não possui o conhecimento necessário para definir uma relação entre a resolução do modelo e o seu desempenho.
Para Liang et al. (2004), a resolução espacial empregada em modelos distribuídos está intrinsicamente relacionada à disponibilidade de base de informações e de memória computacional, uma vez que as distribuições espaciais de precipitação e propriedades do solo são preponderantes na aplicação do modelo.

Nesse contexto, o presente estudo avalia os efeitos da resolução espacial do modelo hidrológico distribuído VIC (sigla em inglês para Variable Infiltration Capacity) para previsão de afluências diárias em cinco usinas hidroelétricas do Rio Uruguai. A área de estudo foi adotada como parte da programação do fundo setorial de pesquisas da Agência Nacional de Energia Elétrica (ANEEL).

\section{MATERIAL E MÉTODOS \\ 2.1 Área de Estudo}

O Rio Uruguai tem origem da confluência dos rios Canoas e Pelotas, este último constituindo parte da fronteira entre os estados de Santa $\mathrm{Ca}$ tarina (SC) e Rio Grande do Sul (RS), e é um dos principais afluentes da bacia do Rio da Prata, segunda maior na América do Sul. A área de estudo está inserida no alto da bacia hidrográfica do Rio Uruguai, definida a jusante pela UHE Foz do Chapecó, que possui área de drenagem com $53.120 \mathrm{~km}^{2}$, englobando outras quatro usinas. As cinco usinas, de jusante para montante, são: Foz do Chapecó, Itá, Machadinho, Campos Novos (rio Canoas, afluente direito) e Barra Grande (rio Pelotas, afluente esquerdo) (Fig. 1). 


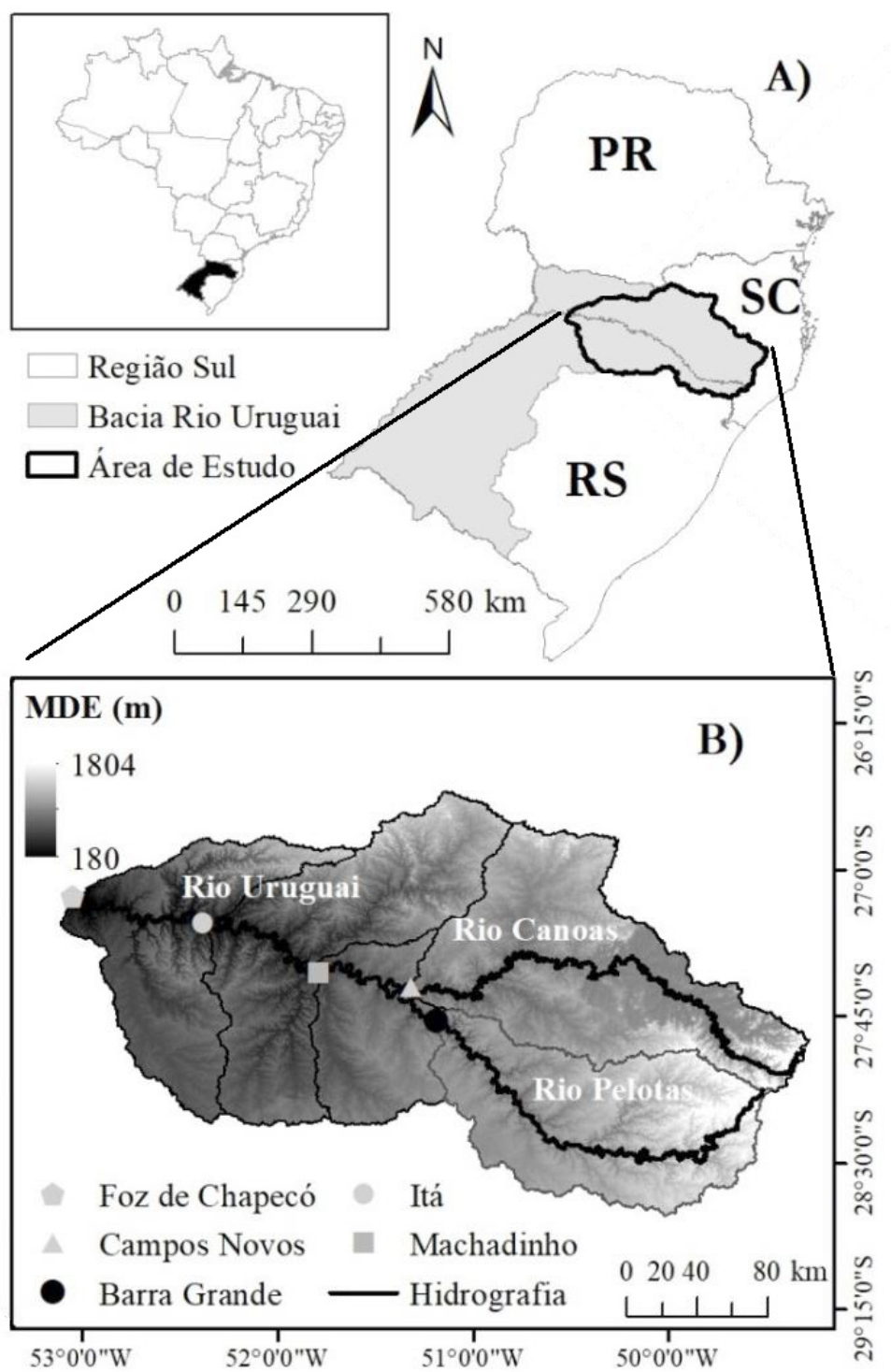

Figura 1 - Localização geográfica da bacia hidrográfica do Rio Uruguai e da área de estudo em território nacional e na região Sul (A); modelo digital de elevação (MDE), hidrografia e localização das usinas hidrelétricas (B).

De acordo com a classificação de Köpen, o clima da região é Cfa (subtropical úmido), com baixas temperaturas nos meses mais frios e precipitação abundante e bem distribuída, não apresentando estação seca ou úmida ao longo do ano (ALVARES et al., 2014). A cobertura vegetal no Alto do Rio Uruguai é formada por floresta ombrófila densa (Mata Atlântica), floresta ombrófila mista (mata de araucária), floresta decidual estacional e campos nativos (estepe). Localizada na formação geológica da Serra Geral, a litologia é formada por rochas basálticas (NARDY et al., 2002) e o solo é predominantemente argiloso com baixa capacidade de infiltração (COLLISCHONN et al., 2005).

O regime de vazões de cheia da área de estudo é caracterizado por não apresentar sazonalidade e apresentar curtos tempos de resposta em relação à precipitação em parte das usinas, devido à presença de relevo montanhoso. As precipi- 
tações anuais registradas variam entre $1500 \mathrm{e}$ 2300-mm, com média igual a 1784-mm. A precipitação é maior na região oeste de Santa Catarina e bem distribuída ao longo do ano; períodos secos e úmidos frequentemente se alternam rapidamente (COLLISCHONN et al., 2005).

A Tabela 1 reúne os dados de área da bacia incremental, volumes dos reservatórios e potência instalada de cada usina, obtidos junto às UHE. Quanto maior o volume útil, maior a presença de erros de balanço hídrico relacionados à leitura da régua limnimétrica na determinação da vazão afluente observada. As usinas hidroelétricas em estudo somam 5.2 GW em capacidade instalada, o equivalente a $5 \%$ da capacidade instalada brasileira.

Tabela 1 - Áreas de drenagem incrementais, volumes dos reservatórios e potência instalada das usinas hidroelétricas (UHE) de Barra Grande, Campos Novos, Machadinho, Itá e Foz do Chapecó.

\begin{tabular}{|c|c|c|c|c|c|}
\hline UHE & $\begin{array}{c}\text { Área incremental } \\
\left(\mathbf{k m}^{2}\right)\end{array}$ & Vol. mín. $\left(\mathrm{hm}^{3}\right)$ & Vol. máx. $\left(\mathrm{hm}^{3}\right)$ & Vol. útil $\left(\mathrm{hm}^{3}\right)$ & Pot. Instalada (MW) \\
\hline Barra Grande & 11889 & 2712 & 4904 & 2193 & 690 \\
\hline Campos Novos & 14426 & 1320 & 1477 & 157 & 880 \\
\hline Machadinho & 5613 & 2283 & 3340 & 1057 & 1140 \\
\hline Itá & 12244 & 5100 & 5100 & 0 & 1450 \\
\hline Foz do Chapecó & 8944 & 1428 & 1502 & 74 & 855 \\
\hline
\end{tabular}

\subsection{Modelo VIC}

O Variable Infiltration Capacity (VIC), desenvolvido para aplicação em grandes bacias hidrográficas (> $10.000 \mathrm{~km}^{2}$ ), é um modelo semi-distribuído que discretiza a área de drenagem em células de grade homogêneas, onde são calculados o balanço hídrico e o balanço energético a cada passo de tempo. A primeira versão do modelo VIC (LIANG, 1994) dividia o perfil do solo em duas camadas (VIC-2L), porém, essa limitação foi removida, dando origem ao modelo VIC-nL (LIANG; $X I E, 2001)$, onde normalmente são empregadas três camadas (VIC-3L), que será utilizada neste estudo. A camada superior é projetada para representar respostas aos eventos de precipitação - podendo gerar escoamento superficial ou ocorrer infiltração - enquanto a camada inferior é utilizada para caracterizar o comportamento do escoamento de base.

A principal característica do modelo VIC é a curva de infiltração variável, que representa a distribuição da capacidade máxima de arma- zenamento de água do solo em cada célula do modelo, determinando a velocidade máxima de infiltração de água e a precipitação efetiva para cada cobertura vegetal da célula, de acordo com a umidade do solo. A variação espacial da capacidade de infiltração do solo é expressa pela distribuição espacial a seguir:

$$
i=i_{m} *\left[1-(1-A)^{\frac{1}{b_{i}}}\right]
$$

onde $i$ e $i_{m}$ são a capacidade de infiltração do solo e a capacidade máxima de infiltração do solo, respectivamente; $A$ é a fração de uma área para a qual a capacidade de infiltração do solo é menor ou igual a $i$; e $b_{i}$ é o parâmetro de forma da capacidade de infiltração do solo, que é a medida da variabilidade espacial da capacidade de infiltração do solo, definida como a quantidade máxima de água que pode ser armazenada na camada superior do perfil do solo. É evidente que se $b_{i}=1$, a capacidade de infiltração de água no solo é uniformemente distribuída. 
Ao final da simulação dos balanços de umidade em cada camada de solo, o modelo de propagação do escoamento (Route), descrito em detalhes por Lohmann et al. (1998), calcula o tempo de concentração para o escoamento atingir a saída de cada célula de grade, bem como o balanço hídrico da onda de cheia na rede hidrográfica. O transporte de água nos canais é descrito pela equação diferencial linear de onda cinemática proposta por Lighthill e Whitham (1955a, 1955b) também conhecida como equação linearizada de Saint-Venant (Eq. 2).

$$
\frac{\partial Q}{\partial t}=D \frac{\partial^{2} Q}{\partial x^{2}}-C \frac{\partial Q}{\partial x}
$$

em que $Q$ é a vazão do escoamento no canal em dimensões de $L^{3} \mathrm{~T}^{-1}, \mathrm{C}$ é a celeridade da onda de cheia no canal em unidades de $\mathrm{LT}^{-1}$ e $\mathrm{D}$ é a difusividade longitudinal da onda de cheia no canal em $L^{2} T^{-1}$.

\subsection{Parametrização do VIC}

A calibração do modelo VIC foi feita por meio do ajuste dos parâmetros mais sensíveis na área de estudo (Tabela 2), os quais foram determinados após análise de sensibilidade. Os parâmetros mais sensíveis em ordem de sensibilidade na área de estudo são: (i) expoente da curva de infiltração variável ( $\left.b_{i}\right)$, o qual controla o particionamento da precipitação entre infiltração e escoamento superficial, onde os valores altos do parâmetro fornecem baixa infiltração, proporcionando, assim, maior escoamento superficial; (ii) espessura da terceira camada do solo (h3), interferindo na transpiração (dependendo da profundidade da raiz) e no escoamento subterrâneo, onde camadas espessas de solo apresentam respostas mais lentas ao escoamento superficial (predominância de escoamento subterrâneo) com alta evapotranspiração, porém resultam na grande retenção de umidade e elevadas vazões de base em períodos secos; (iii) escoamento base máximo na terceira camada de solo (Dm); (iv) fração do escoamento base máximo (Ds) para o início do fluxo base não linear, em que valores altos proporcionam maior escoamento base com menor teor de água na terceira camada de solo; (v) fração do teor de umidade do solo na terceira camada (Ws), onde inicia o escoamento de base não linear. Valores altos de Ws aumentarão a necessidade de água para um aumento de base não linear de rápido aumento, o que tenderá a atrasar os picos de escoamento superficial. Para valores iguais de Ds e Ws, o escoamento base é linear. Além da calibração do modelo VIC, também foi feito o refinamento dos parâmetros do modelo de propagação do escoamento superficial (Route): celeridade da onda de cheia no canal (C) e difusividade longitudinal da onda de cheia no canal (D).

Tabela 2 - Parâmetros dos modelos VIC e Route passíveis de calibração e o significado físico.

\begin{tabular}{|c|c|c|}
\hline Parâmetro & Significado Físico & Amplitude \\
\hline$b_{i}$ & $\begin{array}{c}\text { Parâmetro de curva de infiltração } \\
\text { variável }\end{array}$ & $0,0001-0,400$ \\
\hline $\operatorname{Dm}\left(\mathrm{mm} \mathrm{dia}^{-1}\right)$ & $\begin{array}{l}\text { Fluxo base máximo que ocorre na } \\
\text { terceira camada de solo }\end{array}$ & $0,0-30,0$ \\
\hline Ds (Fração) & $\begin{array}{l}\text { Fração do fluxo base máximo em } \\
\text { que o fluxo base não linear inicia }\end{array}$ & $0,0001-0,999$ \\
\hline Ws (Fração) & $\begin{array}{l}\text { Fração da máxima umidade da } \\
\text { terceira camada de solo em que o } \\
\text { fluxo base não linear inicia }\end{array}$ & $0,0001-0,999$ \\
\hline h3 (m) & Espessura da terceira camada & $0,05-1,50$ \\
\hline $\mathrm{C}\left(\mathrm{m} \mathrm{s}^{-1}\right)$ & Celeridade da onda de cheia & $0,25-3,00$ \\
\hline$D\left(s^{2} m^{-1}\right)$ & $\begin{array}{l}\text { Difusividade longitudinal da onda } \\
\text { de cheia }\end{array}$ & $200-4000$ \\
\hline
\end{tabular}

\subsection{Dados de Entrada e Calibração}

O VIC foi calibrado manualmente utilizando resoluções espaciais de $0,04^{\circ}, 0,08^{\circ}$ e $0,16^{\circ} \mathrm{com}$ total de células de 3211,844 e 249 , respectivamente. As imagens do modelo digital de elevação (MDE) são provenientes da Missão Topográfica Radar Shuttle (SRTM - sigla em inglês) com 
resolução espacial de $90-\mathrm{m}\left(\sim 0,0008^{\circ}\right)$ e foram obtidas no site da Empresa Brasileira de Pesquisa Agropecuária (Embrapa). Na Fig. 2 estão apresentados os MDE's e as declividades para as diferentes resoluções. As resoluções utilizadas neste estudo são provenientes da resolução de referência $\left(0,0008^{\circ}\right)$ por meio do método de agregação, utilizando a função média. Com a redução da resolução, é evidente a perda de informação, tanto no MDE como na declividade.

As variáveis meteorológicas utilizadas para alimentar o modelo VIC correspondem a precipita- ção diária $(\mathrm{mm})$, temperaturas diárias mínima e máxima $\left({ }^{\circ} \mathrm{C}\right)$ e velocidade média diária do vento $\left(\mathrm{m} \mathrm{s}^{-1}\right)$, as quais foram extraídas do modelo numérico de previsão meteorológica Eta-15 km.

O mapeamento das classes de solo foi derivado do mapa de solos da Empresa Brasileira de Pesquisa Agropecuária (Embrapa) e os dados amostrais de solo foram extraídos dos seguintes documentos: Solos do Estado de Santa Catarina (EMBRAPA, 2004) e Folha SH.22 Porto Alegre e parte das Folhas SH.21 Uruguaiana e SI.22 Lagoa Mirim (IBGE, 1986).

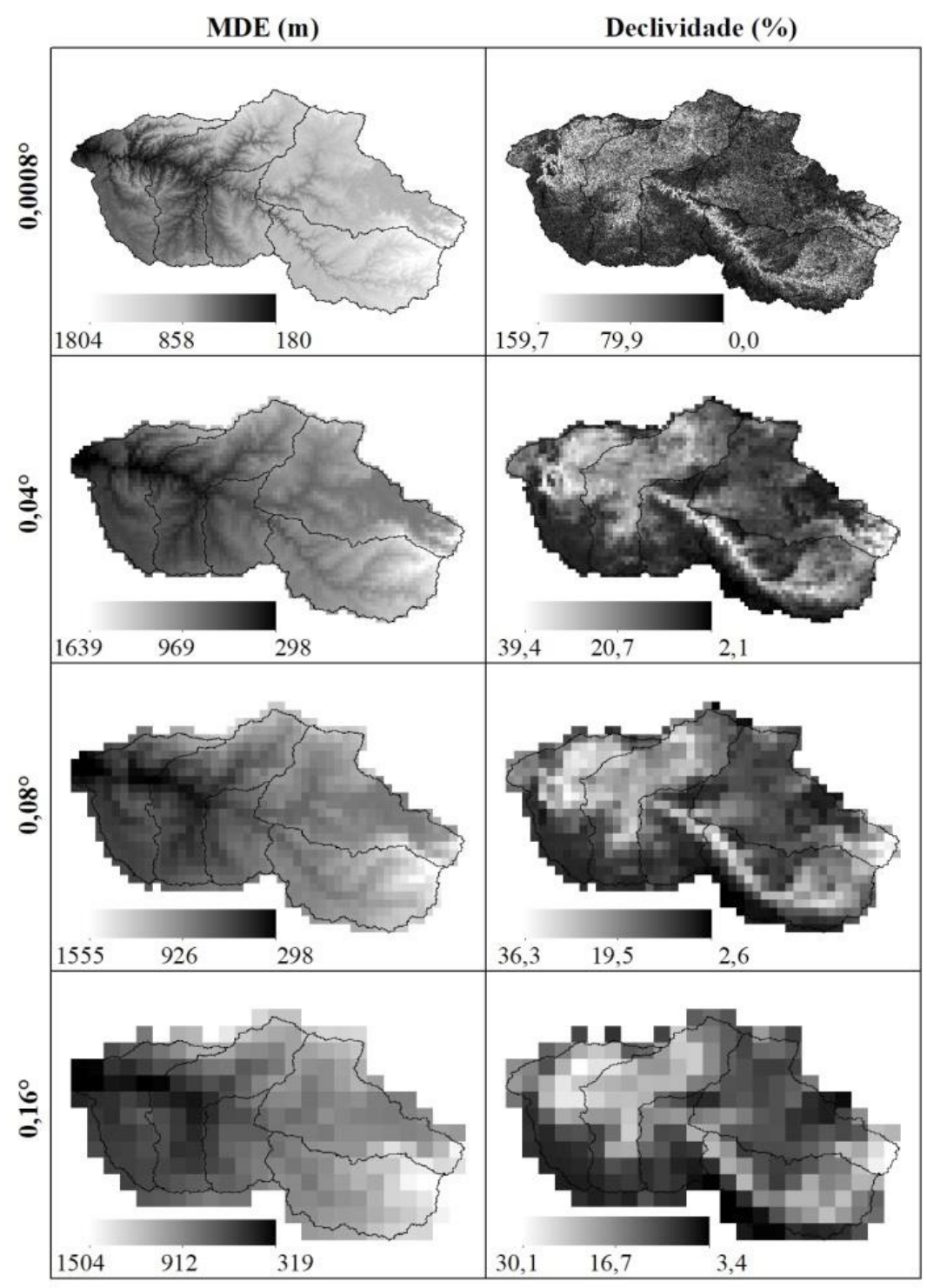

Figura 2 - Modelo digital de elevação (MDE) e declividade da área de estudo para as resoluções $0,0008^{\circ}$ (EMBRAPA), $0,04^{\circ}, 0,08^{\circ}$ e $0,016^{\circ}$. 
Os dados de vazão diária observada permitem a calibração e verificação do modelo por intermédio da comparação com as vazões simuladas. 0 modelo VIC é executado para o período de 2013 a 2017, sendo 2013 o período de aquecimento do modelo, 2014-2015 o período de calibração e 2016-2017 o período de validação. No processo de teste de desempenho foi verificada a aderência das vazões simuladas pelo modelo hidrológico com as vazões afluentes das UHE Barra Grande e Campos Novos e vazões incrementais das UHE Machadinho, Itá e Foz do Chapecó. As vazões foram simuladas isoladamente para a área de drenagem apresentada entre duas UHE consecutivas para permitir isolar a vazão atribuída apenas à transformação chuva-vazão. Tal vazão atribuída apenas à área de drenagem entre dois reservatórios é denominada vazão incremental e é a vazão originada na área de drenagem entre dois reservatórios consecutivos.

\subsection{Desempenho Estatístico}

A avaliação do desempenho da calibração e validação foi realizada a partir de quatro índices estatísticos: coeficiente de determinação $\left(R^{2}\right)$, Nash-Sutcliffe (NASH; SUTCLIFFE, 1970) (NS), Nash-Sutcliffe logarítmico (NS log), percentual de viés (Pbias) e média dos valores absolutos dos desvios (MAD) (Eq. 3, 4, 5, 6 e 7 respectivamente).

$$
\begin{aligned}
& R^{2}=\left\{\frac{\sum_{i=1}^{N}\left(Q_{o i}-\bar{Q}_{o}\right)\left(Q_{s i}-\bar{Q}_{s}\right)}{\sum_{i=1}^{N} \sqrt{\left(Q_{o i}-\bar{Q}_{o}\right)^{2}} * \sqrt{\left(Q_{s i}-\bar{Q}_{s}\right)^{2}}}\right\}^{2} \\
& N S=1-\frac{\sum_{i=1}^{N}\left(Q_{o i}-Q_{s i}\right)^{2}}{\sum_{i=1}^{N}\left(Q_{o i}-\bar{Q}_{o}\right)^{2}} \\
& N S \log =1-\frac{\sum_{i=1}^{N}\left(\log \left(Q_{o i}\right)-\log \left(Q_{s i}\right)\right)^{2}}{\sum_{i=1}^{N}\left(\log \left(Q_{o i}\right)-\log \left(\bar{Q}_{o}\right)\right)^{2}}
\end{aligned}
$$

$$
\begin{aligned}
& P_{\text {bias }}=\left[\frac{\sum_{i=1}^{N}\left(Q_{o i}-Q_{s i}\right)}{\sum_{i=1}^{N} Q_{o i}}\right] * 100 \\
& M A D=\frac{1}{N} * \sum_{i=1}^{N}\left|Q_{o i}-Q_{s i}\right|
\end{aligned}
$$

O símbolo $n$ é o número total de observações, $Q_{o i}$ é a i-ésima observação para a vazão avaliada, $Q_{s i}$ é o i-ésimo valor simulado para a vazão avaliada. A barra sobre a variável representa o valor médio da vazão durante o período analisado. 0 coeficiente de determinação descreve a proporção da variância dos dados observados explicados pelo modelo, que varia entre -1 e 1 , sendo 1 o resultado para o melhor ajuste possível. A eficiência de NS quantifica a diferença entre $1 \mathrm{e}$ o desvio quadrático entre observado e simulado, este relativo à variância das observações. $O$ valor de NS varia de $-\infty$ a 1 , sendo 1 o melhor ajuste possível, pois o desvio terá tendido a zero. Já no NS log, por meio da transformação logarítmica, há maior sensibilidade dos baixos valores de vazão. O percentual de viés $\left(P_{\text {bias }}\right)$ mede a tendência média dos resultados simulados em se apresentarem maiores ou menores que os dados observados (MORASI et al., 2007). Já o MAD traduz o desvio das previsões de vazões em relação aos valores observados. A tendência é que esse valor seja mais elevado para locais com maiores vazões e seja menor para locais com valores menores de vazões. Os resultados desses índices foram utilizados para demonstrar o desempenho do modelo de uma resolução espacial comparativamente em relação ao da outra.

\subsection{Modelo Eta-15km}

O modelo Eta é um modelo meteorológico numérico para representação dos processos físicos e dinâmicos da atmosfera, o qual foi desenvolvido pelo Centro Nacional para Previsões Ambien- 
tais dos Estados Unidos (sigla em inglês NCEP) (BLACK, 1994; MESINGER et al., 1988), e adaptado pelo Centro de Previsão do Tempo e Estudos Climáticos (CPTEC) do Instituto Nacional de Pesquisas Espaciais (INPE), para desenvolvimento de estudos na América do Sul (CHOU; NUNES; CAVALCANTI, 2000).

É um modelo de área limitada em ponto de grade. 0 nome do modelo é derivado da letra grega $\eta$, usada para definir a sua coordenada vertical, a qual é aproximadamente horizontal mesmo em regiões de topografia íngreme. A discretização vertical em níveis geodésicos horizontais é crítica para modelos meteorológicos especialmente quando aplicada em regiões de topografia íngreme (MESINGER et al., 2012). A atmosfera é representada na vertical até o nível de pressão de $25 \mathrm{hPa}$ com 38 níveis (BLACK, 1994). A dinâmica do modelo é desenvolvida na coordenada vertical, sendo mais adequada para regiões montanhosas (CHOU; BUSTAMANTE; GOMES, 2005). O modelo Eta possui diferentes versões que são nomeadas de acordo com a resolução espacial a ser empregada no modelo atmosférico. A versão do modelo Eta utilizada neste trabalho corresponde à resolução espacial de $15 \mathrm{~km}$, que explica a denominação do modelo como Eta-15 km. A resolução espacial a ser adotada foi determinada em $15 \mathrm{~km}$ para reproduzir a resolução encontrada na literatura recente, por exemplo Fan et al. (2017).

\subsection{Previsão de Afluências nas Usinas Hidroelétricas}

Brasil (2016) estabelece critérios e procedimentos de operações para segurança das barragens e controle de cheias. Dentre os critérios e procedimentos, a vazão afluente verificada ou prevista é utilizada para classificação da situação de risco, que possui a seguinte classificação: emergência, alerta, atenção e normal, conforme a Tabela 3.
Tabela 3 - Caracterização da situação de risco de acordo com a vazão prevista ou verificada nas UHE Barra Grande, Campos Novos, Machadinho, Itá e Foz do Chapecó. (Fonte: Brasil, 2016)

\begin{tabular}{|c|c|c|c|}
\hline \multicolumn{4}{|c|}{ Situação de Risco - Vazão $\left(\mathbf{m}^{\mathbf{3}} \mathbf{~ s}^{-\mathbf{1}}\right)$} \\
\hline UHE & Atenção & Alerta & Emergência \\
\hline Barra Grande & $4500-5000$ & $5000-6000$ & $>6000$ \\
\hline Campos Novos & $4000-4500$ & $4500-5000$ & $>5000$ \\
\hline Machadinho & $3000-5000$ & $5000-10000$ & $>10000$ \\
\hline Itá & $4000-6000$ & $6000-10000$ & $>10000$ \\
\hline Foz do Chapecó & $7000-17000$ & $17000-21000$ & $>21000$ \\
\hline
\end{tabular}

Para avaliar o desempenho das previsões de cenários afluentes nas usinas, adotou-se a matriz de contingência (ARONOFF, 1982; CONGALTON, 1991). Existem quatro categorias possíveis (Tabela 4): (i) Verdadeiro Positivo (VP) representa o número de vezes que o modelo previu corretamente, isto é, atingiu ou ultrapassou a vazão de risco em questão; (ii) Falso Positivo (FP) significa o número de vezes que o modelo previu erroneamente a vazão de risco, ou seja, falso alarme para vazão que não ocorreu; (iii) Falso negativo (FN) indica as vezes que o modelo não consegue prever a vazão de risco; e (iv) Verdadeiro Negativo (VN) indica o número de vezes que o modelo prevê corretamente a não ocorrência da vazão de risco.

Tabela 4 - Matriz de contingência. Fonte: ARONOFF (1982); CONGALTON (1991)

\begin{tabular}{|c|c|c|}
\hline \multirow{2}{*}{ Simulação } & \multicolumn{2}{|c|}{ Observação } \\
\cline { 2 - 3 } & Positivo & Negativo \\
\hline Positivo & VP & FP \\
\hline Negativo & FN & VN \\
\hline
\end{tabular}

Três indicadores, incluindo acurácia (ACC), sensibilidade (taxa positiva verdadeira, TPV) e precisão (valor previsto positivo, VPP), foram usados para avaliar a qualidade dos resultados do modelo (Eq. 8,9 e 10, respectivamente):

$A C C=\frac{V P+V N}{V P+V N+F P+F N}$ 
$T P V=\frac{V P}{V P+F N}$

$$
V P P=\frac{V P}{V P+F P}
$$

As situações de risco estabelecidas por Brasil (2016) são referentes à vazão afluente nas usinas. Contudo, o modelo foi calibrado para vazões incrementais nas UHE Machadinho, Itá e Foz do Chapecó. Por conseguinte, para obtenção das vazões de risco incrementais nessas três usinas, subtraíram-se da vazão de risco as vazões defluentes das usinas a montante, como mostrado nas Eq. 11, 12 e 13.

$Q_{M A_{\text {RiscoInc }}}=Q_{M A_{\text {Risco }}}-Q_{C N_{\text {Def }}}-Q_{B G_{\text {Def }}}$

$Q_{I T A_{\text {RiscoInc }}}=Q_{I T A_{\text {Risco }}}-Q_{M A_{\text {Def }}}$

$Q_{F C_{\text {RiscoInc }}}=Q_{F C_{\text {Risco }}}-Q_{I T A_{\text {Def }}}$

Em que Q significa vazão; os subscritos Riscolnc, Risco e Def significam vazões de risco incremental, de risco e defluente, respectivamente; e as abreviações BG, CN, MA, ITA e FC representam as
UHE Barra Grande, Campos Novos, Machadinho, Itá e Foz do Chapecó, respectivamente.

\section{RESULTADOS E DISCUSSÃO}

\subsection{Calibração e Validação}

A Tabela 5 lista os parâmetros que apresentaram sensibilidade significativa à calibração e foram consequentemente calibrados utilizando os modelos VIC e Route para as UHE Barra Grande, Campos Novos, Machadinho, Itá e Foz do Chapecó. A espessura da terceira camada do solo (h3) e a difusividade longitudinal da onda de cheia (D) por sua vez, não se mostraram sensíveis ao processo de calibração dos modelos para todas as áreas de drenagem. A espessura da terceira camada (h3) é a camada de solo mais profunda representada no modelo, porém, ainda assim, pode interferir na transpiração das plantas, dependendo da profundidade das raízes, e no escoamento subterrâneo. A difusividade longitudinal da onda de cheia (D) não foi encontrada sensível devido à configuração encaixada dos vales na bacia que desfavorecem o amortecimento de cheias no vale (o amortecimento de cheias é desempenhado artificialmente pela operação dos reservatórios).

Tabela 5 - Parâmetros calibrados dos modelos VIC e Route para resoluções $0,16^{\circ}, 0,08^{\circ}$ e $0,04^{\circ}$.

\begin{tabular}{|c|c|c|c|c|c|}
\hline Parâmetro & Barra Grande & Campos Novos & Machadinho & Itá & Foz do Chapecó \\
\hline \multicolumn{6}{|c|}{$0,04^{\circ}$} \\
\hline$b$ & 0,382 & 0,075 & 0,075 & 0,384 & 0,300 \\
\hline $\mathrm{Dm}$ & 30,0 & 15,0 & 15,0 & 30,0 & 30,0 \\
\hline Ds & 0,019 & 0,070 & 0,070 & 0,005 & 0,020 \\
\hline Ws & 0,999 & 0,925 & 0,925 & 0,999 & 0,999 \\
\hline $\mathrm{C}$ & 2,87 & 1,52 & 1,71 & 2,95 & 2,93 \\
\hline \multicolumn{6}{|c|}{$0,08^{\circ}$} \\
\hline$b$ & 0,350 & 0,075 & 0,200 & 0,370 & 0,310 \\
\hline $\mathrm{Dm}$ & 30,0 & 15,0 & 15,0 & 30,0 & 30,0 \\
\hline Ds & 0,023 & 0,030 & 0,060 & 0,005 & 0,020 \\
\hline Ws & 0,999 & 0,650 & 0,999 & 0,999 & 0,999 \\
\hline C & 2,81 & 1,45 & 1,33 & 2,90 & 2,88 \\
\hline \multicolumn{6}{|c|}{$0,16^{\circ}$} \\
\hline$b$ & 0,391 & 0,150 & 0,263 & 0,300 & 0,280 \\
\hline $\mathrm{Dm}$ & 30,0 & 15,0 & 2,9 & 30,0 & 30,0 \\
\hline Ds & 0,065 & 0,030 & 0,384 & 0,030 & 0,030 \\
\hline Ws & 0,999 & 0,250 & 0,985 & 0,999 & 0,999 \\
\hline C & 2,10 & 1,50 & 1,35 & 2,93 & 2,84 \\
\hline
\end{tabular}


As áreas de drenagem das UHE Barra Grande e Itá apresentaram maior valor para o parâmetro da curva de infiltração (bi) para todas as resoluções, o que condiz com baixa infiltração e, consequentemente, aumento da precipitação efetiva. De acordo com Fan et al. (2017), o solo da bacia de Barra Grande tem menor capacidade de armazenamento de água, gerando maior escoamento superficial em menor tempo. Em contrapartida, na área a montante da UHE Campos Novos, o parâmetro bi mostrou-se baixo para as maiores resoluções $\left(0,04\right.$ e $\left.0,08^{\circ}\right)$. Esse fato está correlacionado com o perfil do solo e a característica da área de drenagem, a qual apresenta planícies de inundação na região superior, prevalecendo infiltração de água e tornando a resposta de escoamento superficial mais lenta.

Os parâmetros das áreas incrementais das UHE Barra Grande, Itá e Foz do Chapecó apresentaram similaridade para as três resoluções, com valores altos do parâmetro que particiona a precipitação em infiltração e precipitação efetiva (bi) e do escoamento base máximo (Dm). Os tipos de solo que prevalecem nessas áreas - cambissolo e neossolo litólico - sofrem saturação em menor intervalo de tempo, aumentando o escoamento superficial. Além disso, a baixa capacidade de armazenamento favorece o aumento do escoamento base. As diferenças e a variabilidade dos parâmetros para as resoluções espaciais indica que a calibração pode ser específica para cada resolução e os parâmetros não devem ser transferidos por meio da resolução (TROY et al., 2008).

Para avaliação do modelo VIC, utilizou-se como base a vazão diária observada incremental em cada usina hidrelétrica, determinando o desempenho estatístico em relação à vazão diária simulada. Os índices estatísticos resultantes da comparação entre as vazões observadas e simuladas pelo modelo VIC estão contidos na Tabela 6. As duas usinas hidroelétricas a jusante da área de estudo - Foz do Chapecó e Itá - apresentaram melhor desempenho estatístico durante o período de calibração. Dentre as usinas estudadas, ambas possuem o menor volume útil, apresentando, assim, menor erro no balanço hídrico.

Tabela 6 - Índices estatísticos para resoluções espaciais $0,04^{\circ}, 0,08^{\circ}$ e $0,16^{\circ}$ durante os períodos de calibração (20142015) e validação (2016-2017) para as UHE Barra Grande, Campos Novos, Machadinho, Itá e Foz do Chapecó.

\begin{tabular}{|c|c|c|c|c|c|}
\hline \multicolumn{7}{|c|}{ Calibração $0,04^{\circ}$} \\
\hline UHE & $\mathbf{R}^{\mathbf{2}}$ & NS & NS log & Pbias & MAD \\
\hline Barra Grande & 0,541 & 0,527 & 0,561 & $-13,5$ & 236,7 \\
\hline Campos Novos & 0,426 & 0,379 & 0,429 & $-14,2$ & 268,7 \\
\hline Machadinho & 0,448 & 0,423 & 0,372 & $-11,0$ & 109,9 \\
\hline Itá & 0,555 & 0,541 & 0,592 & $-9,2$ & 285,5 \\
\hline Foz do Chapecó & 0,559 & 0,543 & 0,537 & 15,2 & 222,0 \\
\hline UHE & & $0,08^{\circ}$ & & & \\
\hline Barra Grande & 0,552 & 0,542 & 0,570 & $-9,5$ & 231,1 \\
\hline Campos Novos & 0,442 & 0,420 & 0,470 & $-5,4$ & 250,9 \\
\hline Machadinho & 0,452 & 0,426 & 0,371 & $-10,5$ & 110,4 \\
\hline Itá & 0,559 & 0,544 & 0,600 & $-7,3$ & 281,5 \\
\hline Foz do Chapecó & 0,566 & 0,542 & 0,503 & 18,2 & 222,9 \\
\hline & & $\mathbf{0 , 1 6}$ & & & \\
\hline UHE & $\mathbf{R}^{\mathbf{2}}$ & $\mathbf{N S}$ & $\mathbf{N S}$ log & Pbias & MAD \\
\hline Barra Grande & 0,559 & 0,557 & 0,519 & $-6,0$ & 234,0 \\
\hline Campos Novos & 0,438 & 0,409 & 0,448 & $-9,9$ & 259,7 \\
\hline Machadinho & 0,443 & 0,393 & 0,295 & $-17,4$ & 120,8 \\
\hline Itá & 0,552 & 0,541 & 0,584 & $-12,0$ & 289,6 \\
\hline Foz do Chapecó & 0,552 & 0,519 & 0,478 & 21,2 & 224,9 \\
\hline
\end{tabular}

\begin{tabular}{|c|c|c|c|c|c|}
\hline \multicolumn{6}{|c|}{ Validação $0,04^{\circ}$} \\
\hline UHE & $\mathbf{R}^{2}$ & NS & NS log & Pbias & MAD \\
\hline Barra Grande & 0,442 & 0,419 & 0,452 & $-16,3$ & 468,3 \\
\hline Campos Novos & 0,446 & 0,441 & 0,459 & $-3,7$ & 559,2 \\
\hline Machadinho & 0,514 & 0,461 & 0,452 & 3,5 & 260,7 \\
\hline Itá & 0,502 & 0,485 & 0,563 & $-1,5$ & 526,6 \\
\hline Foz do Chapecó & 0,508 & 0,493 & 0,375 & 15,0 & 525,4 \\
\hline \multicolumn{6}{|c|}{$0,08^{\circ}$} \\
\hline UHE & $\mathbf{R}^{2}$ & NS & NS log & Pbias & MAD \\
\hline Barra Grande & 0,419 & 0,405 & 0,527 & $-6,5$ & 468,3 \\
\hline Campos Novos & 0,459 & 0,445 & 0,502 & 7,4 & 559,2 \\
\hline Machadinho & 0,506 & 0,460 & 0,463 & 6,7 & 260,7 \\
\hline Itá & 0,500 & 0,480 & 0,568 & 0,8 & 526,6 \\
\hline Foz do Chapecó & 0,513 & 0,486 & 0,332 & 19,7 & 525,4 \\
\hline \multicolumn{6}{|c|}{$0,16^{\circ}$} \\
\hline UHE & $\mathbf{R}^{2}$ & NS & NS log & Pbias & MAD \\
\hline Barra Grande & 0,405 & 0,402 & 0,424 & $-4,1$ & 468,3 \\
\hline Campos Novos & 0,460 & 0,451 & 0,483 & 2,4 & 559,2 \\
\hline Machadinho & 0,473 & 0,449 & 0,421 & 0,1 & 260,7 \\
\hline Itá & 0,515 & 0,500 & 0,559 & $-4,0$ & 526,6 \\
\hline Foz do Chapecó & 0,498 & 0,465 & 0,312 & 21,8 & 525,4 \\
\hline
\end{tabular}


As usinas com menor desempenho do modelo (Campos Novos e Machadinho) apresentaram escassez de dados ou interferências de reservatórios operados a montante, cujo monitoramento não foi disponibilizado. Segundo Liang et al. (1994), a utilização do modelo VIC em bacias inferiores s 10 mil km², como a área incremental da UHE Machadinho, pode interferir na simulação do balanço hídrico. Além disto, a área incremental da UHE Machadinho possui poucos pontos de amostra de solo (3 pontos), não representando bem a granulometria do solo na área.

Para o período de calibração, a UHE Barra Grande mostrou melhor desempenho para todas as resoluções, sem distinção clara do desempenho entre as resoluções testadas. Três fatores podem ter contribuído para esse comportamento: (i) Barra Grande possui o maior volume útil, o que acarretaria em um maior erro para o balanço hídrico; (ii) a área de drenagem tem declividade acentuada e solo com menor capacidade de armazenamento, gerando, portanto, escoamento superficial em menor tempo; por consequência, a curva da capacidade de infiltração, característica do modelo VIC, teria pouca influência no balanço hídrico; e (iii) a resolução espacial do modelo Eta-15km apresenta viés de baixa para intensidades de precipitação especialmente em locais com efeitos orográficos na precipitação.

O coeficiente Nash-Sutcliffe, empregado com os logaritmos das vazões (NS log), que melhor reflete o desempenho do modelo na simulação de períodos de recessão do hidrograma, mostrou os melhores resultados para a área incremental da UHE Itá, tanto no período de calibração como para va- lidação, variando entre 0,559 e 0,600 e apresentando pouca variação entre as resoluções.

À exceção de Barra Grande, as alterações significativas do desempenho em relação a cada resolução apresentam-se favoravelmente para o aumento da resolução espacial. Observa-se uma inalteração sistemática dos índices de ajuste $\left(R^{2}\right.$, NS e NS log) e uma alteração significativa nos vieses (Pbias). No período de validação do modelo (2016-2017), o desempenho do modelo VIC ficou abaixo de 0,5, exceto para UHE Itá, em que os coeficientes estatísticos $\mathrm{R}^{2}$, NS e NS log se apresentaram maiores ou iguais a 0,5. A média dos valores absolutos dos desvios (MAD) fornece o valor absoluto do erro em escala, de acordo com a dimensão da vazão de cada área incremental.

\subsection{Previsão de Afluências}

A previsão da vazão afluente nas usinas hidrelétricas é fundamental para operação e planejamento da mesma, visando o controle de cheias e a segurança das barragens. Dentre os inúmeros procedimentos de operação e segurança, a vazão afluente prevista é utilizada para classificação de iminentes situações de risco. Nesse sentido, intervalos de vazão são determinados por Brasil (2016) com intuito de avaliar a situação de risco da usina em questão. A Tabela 7 aborda o desempenho do modelo VIC para previsão de vazões nas situações de atenção, alerta e emergência para as UHE Barra Grande, Campos Novos, Machadinho, Itá e Foz do Chapecó durante os anos entre 2014 e 2017 (Tabela 7). 
Tabela 7 - Previsão de contingência (VP, FP, FN e VN) e desempenho (ACC, TPV e VPP) para o período de 2014 a 2017 para as resoluções $0,04^{\circ}, 0,08^{\circ}$ e $0,16^{\circ}$ do modelo VIC para vazões afluentes das UHE Barra Grande, Campos Novos, Machadinho, Itá e Foz do Chapecó.

\begin{tabular}{|c|c|c|c|c|c|c|c|c|c|c|c|c|c|c|c|c|}
\hline \multicolumn{2}{|c|}{$\begin{array}{l}\text { Situação de } \\
\text { Risco }\end{array}$} & \multicolumn{5}{|c|}{ Atenção } & \multicolumn{5}{|c|}{ Alerta } & \multicolumn{5}{|c|}{ Emergência } \\
\hline 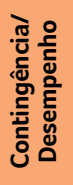 & 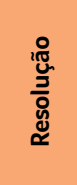 & $\begin{array}{l}\frac{0}{0} \\
\frac{0}{0} \\
\text { U⿺ } \\
\frac{0}{5} \\
\frac{0}{0}\end{array}$ & 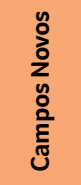 & $\begin{array}{l}\frac{0}{\frac{1}{5}} \\
\frac{5}{0} \\
\frac{0}{0} \\
\frac{\pi}{2}\end{array}$ & $\stackrel{\pi}{=}$ & 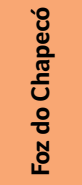 & $\begin{array}{l}\frac{0}{0} \\
\frac{\pi}{\pi} \\
\frac{0}{0} \\
\frac{0}{\pi} \\
\frac{0}{\pi}\end{array}$ & 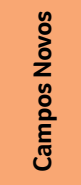 & $\begin{array}{l}\frac{0}{\frac{1}{5}} \\
\frac{5}{0} \\
\frac{\pi}{0} \\
\frac{\pi}{0} \\
\sum\end{array}$ & $\stackrel{\mathbb{I}}{=}$ & 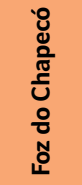 & $\begin{array}{l}\frac{0}{0} \\
\frac{0}{0} \\
\frac{0}{0} \\
\frac{0}{0} \\
\frac{0}{0}\end{array}$ & 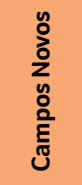 & 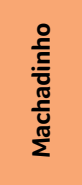 & $\stackrel{\mathbb{Z}}{=}$ & 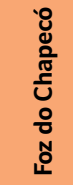 \\
\hline \multirow{3}{*}{ VP } & $0,04^{\circ}$ & 0 & 0 & 33 & 25 & 20 & 0 & 0 & 10 & 12 & 2 & 0 & 0 & 1 & 2 & 1 \\
\hline & $0,08^{\circ}$ & 1 & 0 & 33 & 25 & 20 & 0 & 0 & 10 & 12 & 2 & 0 & 0 & 1 & 2 & 1 \\
\hline & $0,16^{\circ}$ & 1 & 0 & 33 & 25 & 20 & 0 & 0 & 10 & 12 & 2 & 0 & 0 & 1 & 2 & 1 \\
\hline \multirow{3}{*}{$\mathrm{FP}$} & $0,04^{\circ}$ & 0 & 0 & 13 & 22 & 9 & 0 & 0 & 3 & 10 & 1 & 0 & 0 & 1 & 4 & 1 \\
\hline & $0,08^{\circ}$ & 0 & 0 & 12 & 22 & 9 & 0 & 0 & 3 & 10 & 1 & 0 & 0 & 1 & 4 & 1 \\
\hline & $0,16^{\circ}$ & 0 & 0 & 14 & 24 & 8 & 0 & 0 & 3 & 10 & 0 & 0 & 0 & 1 & 4 & 1 \\
\hline \multirow{3}{*}{$\mathrm{FN}$} & $0,04^{\circ}$ & 6 & 1 & 0 & 0 & 0 & 4 & 1 & 0 & 0 & 0 & 0 & 0 & 0 & 0 & 0 \\
\hline & $0,08^{\circ}$ & 5 & 1 & 0 & 0 & 0 & 4 & 1 & 0 & 0 & 0 & 0 & 0 & 0 & 0 & 0 \\
\hline & $0,16^{\circ}$ & 5 & 1 & 0 & 0 & 0 & 4 & 1 & 0 & 0 & 0 & 0 & 0 & 0 & 0 & 0 \\
\hline \multirow{3}{*}{ VN } & $0,04^{\circ}$ & 1394 & 1399 & 1354 & 1353 & 1371 & 1396 & 1399 & 1387 & 1378 & 1397 & 1400 & 1400 & 1398 & 1394 & 1398 \\
\hline & $0,08^{\circ}$ & 1394 & 1399 & 1355 & 1353 & 1371 & 1396 & 1399 & 1387 & 1378 & 1397 & 1400 & 1400 & 1398 & 1394 & 1398 \\
\hline & $0,16^{\circ}$ & 1394 & 1399 & 1353 & 1351 & 1372 & 1396 & 1399 & 1387 & 1378 & 1398 & 1400 & 1400 & 1398 & 1394 & 1398 \\
\hline \multirow{3}{*}{$\mathrm{ACC}$} & $0,04^{\circ}$ & 0,996 & 0,999 & 0,991 & 0,984 & 0,994 & 0,997 & 0,999 & 0,998 & 0,993 & 0,999 & 1,000 & 1,000 & 0,999 & 0,997 & 0,999 \\
\hline & $0,08^{\circ}$ & 0,996 & 0,999 & 0,991 & 0,984 & 0,994 & 0,997 & 0,999 & 0,998 & 0,993 & 0,999 & 1,000 & 1,000 & 0,999 & 0,997 & 0,999 \\
\hline & $0,16^{\circ}$ & 0,996 & 0,999 & 0,990 & 0,983 & 0,994 & 0,997 & 0,999 & 0,998 & 0,993 & 1,000 & 1,000 & 1,000 & 0,999 & 0,997 & 0,999 \\
\hline \multirow{3}{*}{ TPV } & $0,04^{\circ}$ & 0,000 & 0,000 & 1,000 & 1,000 & 1,000 & 0,000 & 0,000 & 1,000 & 1,000 & 1,000 & - & - & 1,000 & 1,000 & 1,000 \\
\hline & $0,08^{\circ}$ & 0,167 & 0,000 & 1,000 & 1,000 & 1,000 & 0,000 & 0,000 & 1,000 & 1,000 & 1,000 & - & - & 1,000 & 1,000 & 1,000 \\
\hline & $0,16^{\circ}$ & 0,167 & 0,000 & 1,000 & 1,000 & 1,000 & 0,000 & 0,000 & 1,000 & 1,000 & 1,000 & - & - & 1,000 & 1,000 & 1,000 \\
\hline \multirow{3}{*}{ VPP } & $0,04^{\circ}$ & - & - & 0,717 & 0,532 & 0,690 & - & - & 0,769 & 0,545 & 0,667 & - & - & 0,500 & 0,333 & 0,500 \\
\hline & $0,08^{\circ}$ & 1,000 & - & 0,733 & 0,532 & 0,690 & - & - & 0,769 & 0,545 & 0,667 & - & - & 0,500 & 0,333 & 0,500 \\
\hline & & 1,000 & - & 0,702 & 0,510 & 0,714 & - & - & 0,769 & 0,545 & 1,000 & - & - & 0,500 & 0,333 & 0,500 \\
\hline
\end{tabular}

A acurácia do modelo (ACC) representa a proporção de acertos da previsão quanto a ambos os lados da restrição operativa. Todas as usinas mostraram acurácia superior a $98 \%$ para todas as resoluções. A resolução espacial do modelo demonstrou efeito sobre a acurácia limitada apenas ao nível de atenção.

A sensibilidade (TPV) avalia o número de verdadeiros positivos (VP) obtidos pelo modelo em relação ao número de positivos observados. As UHE Barra Grande e Campos Novos mostraram baixa ou nenhuma sensibilidade para previsão de vazões nas situações de atenção e alerta. Já, ressalta-se que, entre o período estudado (20142017), não houve ocorrência de vazões superiores à vazão de emergência para ambas as usinas. Em contrapartida, para todas as situações de risco, as usinas a jusante (Machadinho, Itá e Foz do Chapecó) apresentaram sensibilidade 100\% para todas as resoluções.

A precisão (VPP) analisa o número de verdadeiros positivos (VP) obtidos pelo modelo em relação ao número de positivos previstos. Para as UHE Machadinho, Itá e Foz do Chapecó, nas situações de atenção e alerta, o modelo mostrou precisão variando entre 0,51 e 1,00, em que a menor resolução $\left(0,16^{\circ}\right)$ mostrou a menor precisão e mais precisão de maneira difusa entre as resoluções. Já na situação de emergência, as UHE Machadinho e Foz do Chapecó mostraram precisão de 0,5 para todas as resoluções e a UHE Itá apresentou precisão de 0,333 , igualmente para todas as resoluções. 


\section{CONCLUSÃO}

A resolução espacial do modelo hidrológico VIC acarreta o aumento de desempenho de previsões de vazão diária e dos riscos de cheias de forma heterogênea sobre a bacia do Rio Uruguai. As usinas de jusante (Machadinho, Itá e Foz do Chapecó) foram favorecidas tanto na previsão diária de vazão afluente, aplicada para a geração, como na identificação de cheias. Quanto à previsão diária, observou-se a tendência de melhores previsões com relação ao refinamento da resolução espacial do modelo de forma diferenciada entre as usinas devido ao maior erro de balanço hídrico e maiores erros de previsão pluviométrica nas usinas de montante.

Os efeitos da resolução espacial do modelo VIC foram percebidos parcialmente entre as métricas de desempenho da previsão de cheias, estas classificadas em cada usina conforme a classificação do Inventário de Restrições Operativas Hidráulicas do Aproveitamento Hidroelétrico $(\mathrm{ROH})$ do ONS. O efeito da resolução espacial do modelo VIC na previsão de riscos de cheias demonstra sensibilidade invariável igual a $100 \%$, uma vez que não houve falsos negativos. A métrica de precisão variou de 51 a 100\%, uma vez que houve poucos ou nenhum falso positivo. Ambas as métricas não apresentaram tendência significativa em relação à resolução espacial para todas as usinas. Todas as usinas mostraram acurácia mínima de 98,3\% e de forma crescente em relação à resolução espacial do modelo. Essa acurácia demonstra um alto nível de acerto do modelo como um todo, para ocorrência do risco de cheia (positivo verdadeiro) e falta do risco (negativo verdadeiro).

Embora este trabalho tenha adotado resoluções espaciais acima das normalmente praticadas no estado-da-técnica, o maior refinamento da resolução espacial do modelo hidrológico não levou em todos os casos a maiores desempenhos do modelo hidrológico. Para que uma maior resolução espacial do modelo hidrológico se reflita em maior desempenho, é necessário que esta se reflita antes em uma maior depreensão de parâmetros e dados da bacia hidrográfica até a resolução mais fina utilizada, o que não ocorre homogeneamente nesta bacia. Portanto, a heterogeneidade de dados foi fator limitante para os ganhos do refinamento da resolução espacial do modelo.

\section{AGRADECIMENTOS}

Os autores agradecem à Agência Nacional de Energia Elétrica pelos recursos destinados ao projeto de pesquisa e desenvolvimento intitulado Otimização da Geração Integrada ao Controle de Cheias nas Usinas da Bacia do Rio Uruguai, solicitados por intermédio das companhias de geração hidroelétrica Baesa, Enercan, Ceran, Engie, Paulista Lageado e Foz do Chapecó.

\section{CONTRIBUIÇÃO DOS AUTORES}

Todos os autores contribuíram de forma igualitária.

\section{REFERÊNCIAS}

ALVARES, C. A. et al. Koppen's climate classification map for Brazil. Meteorologische Zeitschrift, v. 22, n. 6, p. 711-728, 2014. https://doi.org/10.1127/0941-2948/2013/0507

ANDERSEN, J. et al. Use of remotely sensed precipitation and leaf area index in a distributed hydrological model. Journal of Hydrology, v. 264, p. 34-50, 2002. https://doi.org/10.1016/ S0022-1694(02)00046-X

ARONOFF, S. Classification Accuracy: A User Approach. Photogrammetric Engineering and Remote Sensing, v. 48, n. 8, p. 1299-1307, 1982

BEVEN, K. How far can we go in distributed hydrological modelling ? Hydrology and Earth System Sciences, v. 5, n. 1, p. 1-12, 2001. https://doi.org/10.5194/hess-5-1-2001

BLACK, T. L. The new NMC mesoscale Eta model: description and forecast axamples. Weather and Forecasting, v. 9, n. 2, p. 265278, 1994.

Brasil (2016). Inventário das Restrições Operativas Hidráulicas dos Aproveitamentos Hidroelétricos. Operador Nacional do Sistema. Rio de Janeiro. 2016. 
CHOU, C.; NUNES, A. M. B.; CAVALCANTI, I. F. A. Extended range forecasts over South America using the regional eta model. Journal of Geophysical Research, v. 105, n. D8, p. 10147-10160, 2000.

CHOU, S. C.; BUSTAMANTE, J. F.; GOMES, J. L. Nonlinear Processes in Geophysics Evaluation of Eta Model seasonal precipitation forecasts over South America. n. 2001, p. 537-555, 2005.

COLLISCHONN, W. et al. Forecasting River Uruguay flow using rainfall forecasts from a regional weather-prediction model. Journal of Hydrology, v. 305, n. 1-4, p. 87-98, 2005. https://doi. org/10.1016/J.JHYDROL.2004.08.028

COLLISCHONN, W. et al. Medium-range reservoir inflow predictions based on quantitative precipitation forecasts. Journal of Hydrology, v. 344, p. 112-122, 2007. https://doi.org/10.1016/j. jhydrol.2007.06.025

COLLISCHONN, W. et al. The MGB-IPH model for large-scale rainfall - runoff modelling The MGB-IPH model for large-scale rainfall - runoff modelling. Hydrological Sciences Journal, v. 52, p. 878-894, 2010. https://doi.org/10.1623/hysj.52.5.878

COLLISCHONN, W.; TUCCI, C. E. M. Simulação Hidrológica de Grandes Bacias. Revista Brasileira de Recursos Hídricos, v. 6, n. 1, p. 95-118, 2001.

CONGALTON, R. G. A Review of Assessing the Accuracy of Classifications of Remotely Sensed Data. Remote Sens. Environ., v. 46, p. 35-46, 1991.

DALSENO, T. C.; ZAMBON, R. C.; BARROS, M. T. L.; YEH, W. Evaluation of Monthly Inflow Forecasting Models for the Planning and Management of the Brazilian Hydropower System. World Environmental and Water Resources Congress 2017. Anais... https:///doi.org/10.1061/9780784480601.045

EMPRESA BRASILEIRA DE PESQQUISA AGROPECUÁRIA - EMBRAPA. Solos do Estado de Santa Catarina. Rio de Janeiro: EMBRAPA, 2004.

FAN, F. M. et al. Evaluation of upper Uruguay river basin (Brazil) operational flood forecasts. Brazilian Journal of Water Resources, v. 22, n. 37, 2017. https://doi.org/10.1590/23180331.0217160027

GUILHON, L. G. F.; ROCHA, V. F.; MOREIRA, J. C. Comparação de Métodos de Previsão de Vazões Naturais Afluentes a Aproveitamentos Hidroelétricos. Revista Brasileira de Recursos Hídricos, v. 12, n. 3, p. 13-20, 2007.

HADDELAND, I.; MATHEUSSEN, B. V; LetTenMAIER, D. P. Influence of spatial resolution on simulated streamflow in a macroscale hydrologic model. Water Resources Research, v. 38 , n. 7, p. 1-10, 2002.

INSTITUTO BRASILEIRO DE GEOGRAFIA E ESTATÍSTICA - IBGE. Folha SH.22 Porto Alegre e partes das Folhas SH.21 Uruguaiana e SI.22 Lagoa Mirim. Rio de Janeiro: IBGE, 1986.
LIANG, X. Two-layer variable infiltration capacity land surface representation for general circulation models. Water Resources Serie, n. 140, p. 208, 1994.

LIANG, X.; GUO, J.; LEUNG, L. R. Assessment of the effects of spatial resolutions on daily water flux simulations. Journal of Hydrology, v. 298, p. 287-310, 2004. https://doi.org/10.1016/j. jhydrol.2003.07.007

LIANG, X.; XIE, Z. A new surface runoff parameterization with subgrid-scale soil heterogeneity for land surface models. Advances in Water Resources, v. 24, p. 1173-1193, 2001. https:// doi.org/10.1016/S0309-1708(01)00032-X

LIGHTHILL, M. J.; WHITHAM, G. B. On kinematic waves I. Flood movement in long rivers. Proceedings of Royal Society London, $v$. 229, p. 281-316, 1955a. https://doi.org/10.1098/rspa.1955.0088

LIGHTHILL, M. J.; WHITHAM, G. B. On kinematic waves II. A theory of traffic flow on long crowded roads. Proceedings of Royal Society London, v. 229, p. 317-345, 1955b. https://doi.org/10.1098/ rspa.1955.0089

LOHMANN, D. et al. Regional scale hydrology: I. Formulation of the VIC-2L model coupled to a routing model. Hydrological Sciences Journal, v. 43, n. 1, p. 131-141, 1998. https://doi. org/10.1080/02626669809492107

MESINGER, F. et al. The Step-Mountain Coordinate: Model Description and Performance for Cases of Alpine Lee Cyclogenesis and for a Case of an Appalachian Redevelopment. Monthly Weather Review, v. 116, n. 7, p. 1493-1518, 1988. https://doi. org/10.1175/1520-0493(1988)116<1493:TSMCMD>2.0.CO;2

MESINGER, F. et al. An upgraded version of the Eta model. Meteorology and Atmospheric Physics, v. 116, n. 3-4, p. 63-79, 2012. https://doi.org/10.1007/s00703-012-0182-z

MORASI, D. N. . et al. Model Evaluation Guidelines for Systematic Quantification of Accuracy in Watershed Simulations. Transactions of the ASABE, v. 50, n. 3, p. 885-900, 2007. https:// doi.org/10.13031/2013.23153

MOREDA, F. et al. Parameterization of distributed hydrological models: learning from the experiences of lumped modeling. Journal of Hydrology, v. 320, p. 218-237, 2006. https://doi. org/10.1016/j.jhydrol.2005.07.014

NARDY, A. J. R. et al. Geologia e Estratigrafia da Formação Serra Geral. Revista Geociências, v. 21, p. 15-32, 2002.

NASH, J. E.; SUTCLIFFE, J. V. River Flow Forecasting Through Conceptual Models Part I - A Discussion of Principles*. Journal of Hydrology, v. 10, n. 3, p. 282-290, 1970. https://doi. org/10.1016/0022-1694(70)90255-6

TROY, T. J.; WOOD, E. F.; SHEFFIELD, J. An efficient calibration method for continental-scale land surface modeling. Water Resources Research, v. 44, p. 1-13, 2008. https://doi. org/10.1029/2007WR006513 